Check for updates

Cite this: Phys. Chem. Chem. Phys., 2017, 19, 25796

DOI: $10.1039 / c 7 c p 90209 c$

rsc.li/pccp

\section{Correction: Observation of crystalline changes of titanium dioxide during lithium insertion by visible spectrum analysis}

\author{
Inho Nam, $\dagger^{a}$ Jongseok Park, $\dagger^{a}$ Soomin Park, ${ }^{a}$ Seongjun Bae, ${ }^{a}$ Young Geun Yoo, ${ }^{a}$ \\ Jeong Woo Han ${ }^{\mathrm{b}}$ and Jongheop Yi*a
}

Correction for 'Observation of crystalline changes of titanium dioxide during lithium insertion by visible spectrum analysis' by Inho Nam et al., Phys. Chem. Chem. Phys., 2017, 19, 13140-13146.

The authors wish to correct the Acknowledgements in their published article. The amended Acknowledgements are shown below.

This work was supported by the National Research Foundation of Korea (NRF) grant funded by the Korean government (MEST) (NRF-2016R1E1A1A01942936) and the Supercomputing Center/Korea Institute of Science and Technology Information with supercomputing resources including technical support (KSC-2015-C2-035).

The Royal Society of Chemistry apologises for these errors and any consequent inconvenience to authors and readers.

\footnotetext{
${ }^{a}$ School of Chemical and Biological Engineering, WCU Program of C2E2, ICP, Seoul National University, Seoul 08826, Republic of Korea. E-mail: jyi@snu.ac.kr

${ }^{b}$ Department of Chemical Engineering, University of Seoul, Seoul 02504, Republic of Korea

$\dagger$ These authors contributed equally to this work.
} 